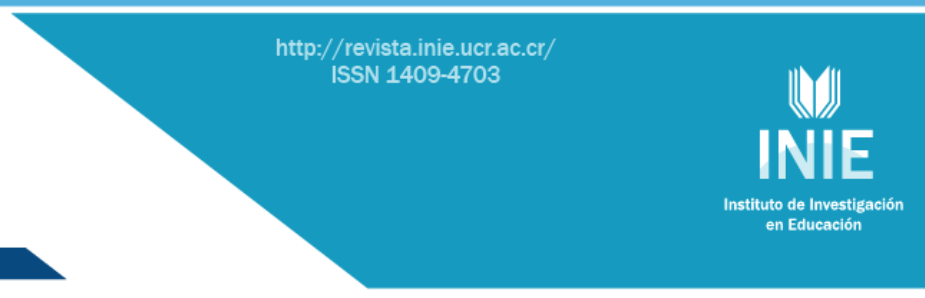

DE LA INTEGRACIÓN A LA INCLUSIÓN: EVOLUCIÓN Y CAMBIO EN LA MENTALIDAD DEL ALUMNADO UNIVERSITARIO DE EDUCACIÓN ESPECIAL EN UN CONTEXTO UNIVERSITARIO ESPAÑOL

FROM INTEGRATION TO INCLUSION: EVOLUTION AND CHANGE IN THE MENTALITY OF UNIVERSITY STUDENTS OF SPECIAL EDUCATION IN SPANISH UNIVERSITY CONTEXT

Volumen 13, Número 3

Setiembre - Diciembre pp. 1-27

Este número se publicó el 30 de setiembre de 2013

Juan José Leiva Olivencia

Revista indizada en REDALYC, $\underline{\text { SCIELO }}$

Revista distribuida en las bases de datos:

CATÁLOGO DE LATINDEX, IRESIE, CLASE, DIALNET, DOAJ, E-REVIST@S, SHERPA/ROMEO, QUALIS, MIAR

Revista registrada en los directorios:

ULRICH'S, REDIE, RINACE, OEI, MAESTROTECA, PREAL, CLASCO 


\title{
DE LA INTEGRACIÓN A LA INCLUSIÓN: EVOLUCIÓN Y CAMBIO EN LA MENTALIDAD DEL ALUMNADO UNIVERSITARIO DE EDUCACIÓN ESPECIAL EN UN CONTEXTO UNIVERSITARIO ESPAÑOL

\author{
FROM INTEGRATION TO INCLUSION: EVOLUTION AND CHANGE IN THE MENTALITY \\ OF UNIVERSITY STUDENTS OF SPECIAL EDUCATION IN SPANISH UNIVERSITY \\ CONTEXT
}

\begin{abstract}
Juan José Leiva Olivencia ${ }^{1}$
Resumen. El presente artículo pretende suscitar la reflexión pedagógica sobre el cambio de mentalidad que se ha producido en un grupo estudiantil de la carrera de Educación Especial en la Facultad de Educación de la Universidad de Málaga (Andalucía, España). El objetivo era estudiar el sentido y el significado de una educación que está evolucionando con claridad desde una perspectiva pedagógica de integración hacia una de inclusión social y educativa. Para recoger los datos se realizaron entrevistas semiestructuradas y autoinformes a 70 estudiantes, posteriormente, se desarrolló un análisis cualitativo de contenido. Como resultado del análisis se pone de relieve que esta evolución conceptual resulta sumamente positiva en el ámbito universitario y que es necesario articular nuevos discursos y prácticas docentes que proyecten e iluminen la idea de que la educación inclusiva es un proceso de cambio permanente.
\end{abstract}

Palabras clave: EDUCACIÓN INCLUSIVA, EDUCACIÓN ESPECIAL, FORMACIÓN INICIAL DEL PROFESORADO, CULTURA DE LA DIVERSIDAD, ESPAÑA.

\begin{abstract}
This article pretends to raise pedagogical reflection about the change of mindset that has taken place in a student group from the program of special education in the Faculty of Education of the University of Malaga (Andalusia, Spain). The objective was to study the meaning and significance of education that is evolving with clearly from a pedagogical perspective of integration toward social and educational inclusion. To collect data on semi-structured interviews and personal reports were conducted with 70 students; afterwards, a qualitative analysis of content was developed. Among the results founded it highlights that this conceptual evolution is extremely positive in the university context and that it is necessary to articulate new discourses and teaching practices that project and illuminate the idea that inclusive education is a process of permanent change.
\end{abstract}

Key words: INCLUSIVE EDUCATION, SPECIAL EDUCATION, INITIAL TEACHER TRAINING, CULTURE OF DIVERSITY, SPAIN.

\footnotetext{
1 Profesor del Departamento de Didáctica y Organización Escolar, Facultad de Ciencias de la Educación, Universidad de Málaga, España.
}

Dirección electrónica: juanleiva@uma.es

Artículo recibido: $1^{\circ}$ de abril, 2013

Aprobado: 12 de setiembre, 2013 


\section{Introducción}

Los sistemas educativos actuales vienen afrontando en las últimas décadas el reto de la calidad y la excelencia educativa en una era compleja y en permanente cambio social (Pérez, 2012). Así, hablar de inclusión educativa y social es indagar en una formación de calidad y excelencia en todas las instituciones formativas del siglo XXI, y ello implica una formación que también debe ser inclusiva, sustentadora de valores, principios y convicciones democráticas y solidarias.

Ahora bien, no se puede olvidar que la excelencia también depende del grado de inclusión de todo el alumnado, también de aquel que puede mostrar en algún momento de su escolarización algún tipo de necesidad educativa específica (Escudero y Martínez, 2011). Es decir, la calidad y la excelencia académica deben atender a parámetros de equidad y de atención a la diversidad para la construcción de una escuela más igualitaria, que ofrezca realmente igualdad de oportunidades para la mejor cohesión y progreso social. En este punto, nadie duda de la necesidad educativa de un cambio de paradigma, en la mentalidad y en la propia identidad de los centros educativos en relación a la educación inclusiva, que todavía persiste identificándose como una educación especial que aboga por la integración y no por la inclusión (López, 2004 y 2006; Stainback y Stainback, 2001).

Además, en el caso concreto de las políticas de atención a la diversidad emprendidas por las administraciones educativas autonómicas del Estado Español, si bien desde una perspectiva teórica se opta por la inclusión; la práctica, normas y procedimientos de escolarización del alumnado con necesidades educativas especiales puede llegar a provocar procesos de segregación de algunos de ellos en centros de educación especial, lo cual va en detrimento de la inclusión social y pedagógica (Susinos y Parrilla, 2008), no solamente defendida por profesionales de la educación sino por muchas familias e instancias sociales (Echeita, 2006). Es decir, a nivel teórico e incluso de normativa vigente se defiende la inclusión educativa (MECD, 2006), pero la traducción burocrática y administrativa de los procesos de escolarización y de organización escolar en ocasiones desvirtúa el sentido y esencia de los principios pedagógicos de la inclusión.

\section{La Educación inclusiva: conceptualización y fundamentos teóricos}

Educar en la diferencia supone una actitud de valoración positiva hacia la comunicación e interacción entre personas diferentes, y hacia la comprensión de lo diverso 
como un factor de aprendizaje positivo y necesario en las actuales organizaciones escolares. Ahora bien, el reto es complejo, y en la actualidad defendemos la idea de que la educación inclusiva se encuentra entre el deseo de ser una propuesta educativa crítica, y la realidad de encontrarse en una encrucijada de caminos que van desde la segregación encubierta del alumnado con necesidades educativas especiales a la falta de profesionales especializados, así como la escasa formación en educación inclusiva de todo el profesorado y la comunidad educativa.

En este sentido, todavía existe un enfoque curricular compensatorio o deficitario en el tratamiento educativo del alumnado con necesidades educativas especiales, lo cual es a todas luces reprochable no solamente por el mundo académico comprometido, sino también por los profesionales de la educación y la orientación educativa, así como por las familias que viven con malestar cómo el sistema educativo no es capaz de garantizar una atención inclusiva y de calidad -en igualdad- a sus hijos e hijas (Ainscow, 2001; Arnaiz, 2003).

En el ámbito de la formación universitaria, en el caso concreto de las Facultades de Ciencias de la Educación, también es digno de mencionar la necesidad de profundizar en la concepción pedagógica inclusiva no sólo del alumnado, sino también del profesorado. Las instituciones universitarias de educación deben ser motores de cambio educativo y de transformación de mentalidades sociales para la mejora y el progreso de la sociedad y la ciencia.

En el caso concreto de la mención de Educación Inclusiva que algunas universidades andaluzas y españolas han incorporado en los planes de estudio de los nuevos grados de Educación Primaria (donde se encuentra la especialidad de Educación Inclusiva, anteriormente denominada Educación Especial), es posible que sea necesario un mayor nivel de especialización, ya que no podemos olvidar la importancia de promover una formación inclusiva en todas las materias, lo cual resulta un eje vertebrador de una docencia comprometida y crítica en tiempos de incertidumbre social y económica como la que estamos viviendo en España desde el año 2008 hasta la actualidad. Así pues, no podemos negar que muchos docentes universitarios defiendan una perspectiva abierta, holística y compleja de la educación inclusiva, partiendo de la propia definición que hace la UNESCO (2005, p.14):

La educación inclusiva puede ser concebida como un proceso que permite abordar y responder a la diversidad de las necesidades de todos los educandos a través de una mayor participación en el aprendizaje, las actividades culturales y comunitarias y 
reducir la exclusión dentro y fuera del sistema educativo. Lo anterior implica cambios y modificaciones de contenidos, enfoques, estructuras y estrategias basados en una visión común que abarca a todos los niños en edad escolar y la convicción de que es responsabilidad del sistema educativo regular educar a todos los niños y niñas. El objetivo de la inclusión es brindar respuestas apropiadas al amplio espectro de necesidades de aprendizaje tanto en entornos formales como no formales de la educación. La educación inclusiva, más que un tema marginal que trata sobre cómo integrar a ciertos estudiantes a la enseñanza convencional, representa una perspectiva que debe servir para analizar cómo transformar los sistemas educativos y otros entornos de aprendizaje, con el fin de responder a la diversidad de los estudiantes. El propósito de la educación inclusiva es permitir que los maestros y estudiantes se sientan cómodos ante la diversidad y la perciban no como un problema, sino como un desafío y una oportunidad para enriquecer las formas de enseñar y aprender.

En este punto, compartimos con el profesor López (2004 y 2006) quien plantea la necesidad de formar a los futuros docentes, los docentes del siglo XXI, en una cultura de la diversidad que fundamenta la educación inclusiva, su concepción y sus prácticas didácticas. $Y$ es que plantea que, en un sistema escolar moderno, el alumnado, lejos de ser un mero receptor pasivo de información y normas, aprende de manera activa explorando, seleccionando y transformando el material de aprendizaje. Dentro de esta visión moderna está el pensamiento del constructivismo piagetiano, por un lado, que supone que el alumnado necesita de un entorno que le estimule para la resolución de problemas y les permita desarrollar y practicar sus esquemas mentales $\mathrm{y}$, por otro, el pensamiento vygotskyano, que subraya que el hecho de que el alumno sea capaz de aprender por su cuenta es más un resultado del proceso educativo que un punto de partida del mismo. Esto supone un cambio no sólo de mentalidad para el futuro profesorado, sino que en su vivencia como alumnado puede suponer una nueva mirada a su propio ser y estar en aulas universitarias que también promuevan la inclusión y el aprendizaje cooperativo como elementos clave.

Vivimos en un mundo en constante cambio, especialmente en el ámbito educativo. Existe una preocupación cada vez mayor por mejorar el capital humano y el bienestar social, y ahí el papel de la educación es de suma importancia, ya que invertir en educación no es un 
gasto sino una inversión de futuro, de crecimiento social y desarrollo económico (Verdugo, 2009). Los procesos de transformación que hace unas décadas podían alargarse durante años se suceden ahora vertiginosamente gracias a la rapidez e inmediatez que nos proporcionan las TIC. Lo que hoy es nuevo, mañana resulta anticuado. Así pues, nos encontramos con un nuevo mapa político, social y cultural que se dibuja como inestable por su naturaleza altamente variable. La escuela, como institución integrante de la sociedad y reflejo de ella, no puede permanecer ajena al cambio de escenario y se ve obligada a renovarse para dar respuesta a los desafíos que la sociedad del siglo XXI le presenta. La creciente proporción de alumnado extranjero por clase, la disparidad de conocimientos, de culturas, de estilos de vida... (todo ello sin disminución en la ratio de discentes por aula) dan forma a un rompecabezas de difícil solución. Por un lado, la sociedad nos exige una mayor preparación académica para afrontar una realidad cada vez más compleja; por otro, la escuela intenta hacer frente a dicha complejidad con estrategias que no siempre se corresponden con la diversidad imperante en las aulas.

Resulta evidente que este panorama educativo genera nuevas necesidades de formación en los docentes encargados de desarrollar prácticas educativas capaces de responder con garantías de éxito ante el desafío de una nueva educación para una nueva sociedad. Son numerosos los autores que coinciden en señalar que el profesorado es el agente clave para el cambio de cualquier sistema educativo y que de su actuación depende el éxito de todo programa de innovación educativa. De ahí que la formación de los docentes se convierta en un aspecto de especial atención dentro de la provisión educativa.

Recientemente, en el contexto de la Unión Europea, con la reforma de los planes de estudio universitarios para su adaptación al EEES (Espacio Europeo de Educación Superior), hemos presenciado la aparición de nuevos módulos curriculares adaptados a las nuevas necesidades de formación. En el caso del grado de Educación Primaria, resulta especialmente significativa en algunas Facultades de Ciencias de la Educación la aparición de la asignatura "Hacia una escuela inclusiva: modelos y prácticas", ya que supone el reconocimiento institucional a la necesidad de que todo docente se forme en el manejo de las estrategias de desarrollo de prácticas de carácter inclusivo. La escuela inclusiva se presenta como respuesta no sólo al escenario educativo actual, sino también al social. Debemos recordar que la escuela es el primer lugar donde niños y niñas de diferentes entornos sociales se encuentran y por tanto, el lugar idóneo para aprender a convivir en la 
diversidad. La experiencia nos enseña que si no aprendemos a valorar las diferencias en el aula, difícilmente aprenderemos a hacerlo en una sociedad con un marcado carácter elitista, competitivo y materialista. La escuela inclusiva, en tanto que se propone erradicar cualquier forma de discriminación y promover la cohesión social, se puede concebir como la esperanza de transformación de la sociedad hacia un futuro más justo, más equitativo y, en definitiva, más humana.

El origen del movimiento inclusivo se sitúa en los países anglosajones, dentro del marco de la educación especial. Es por ello que tradicionalmente se ha asociado a la educación inclusiva con una educación sólo para el alumnado con necesidades específicas de atención educativa, cuando la realidad es que la escuela inclusiva se concibe como una escuela para todos y todas.

Si bien son diversas las definiciones sobre educación inclusiva que podemos encontrar en la literatura, en un intento por sintetizar sus bases podríamos decir que la escuela inclusiva reconoce la diversidad como valor, de modo que todo el mundo tiene cabida en ella en situación de igualdad. El reconocimiento de las diferencias individuales nos lleva al reconocimiento de la diversidad, no sólo cognitivo sino también emocional y del mundo de valores. Así, desde el punto de vista de la inclusión social y educativa, la diversidad es reconocimiento de diferencias y valoración de ellas, esto es, necesidad de aprovechamiento de las mismas para todo el alumnado y la comunidad educativa, y no una lacra perturbadora de la convivencia escolar.

Quizá esta última idea sea una de las más importantes: la diversidad se valora; esto es, no se considera deficiencia, carencia o desviaciones de un perfil normativo. Si comparamos a un individuo con un perfil normativo (y le pedimos que se adapte a él), no estaremos reconociendo sus peculiaridades, sino la desigualdad. Entonces surge la jerarquía, porque hay quien supera la norma y quien queda debajo de ella, diciéndose de estos últimos que han fracasado (porque no tienen el "nivel" requerido). Por tanto, la desigualdad surge cuando se institucionalizan las diferencias como deficiencias, y es este etiquetado de deficiencia el que justifica la exclusión. Efectivamente, la desigualdad va más allá de la escuela, de los métodos pedagógicos y de los procesos de evaluación que se emplean en las aulas (Miles y Singal, 2009). La desigualdad se encuentra enmarcada en la mentalidad colectiva y es necesario romper con toda una serie de prejuicios y estereotipos hacia los niños y niñas con discapacidad, así como con sus familias (López, 2004). 
La UNESCO (2009) establece que la educación inclusiva se basa en el derecho de todo el alumnado a recibir una educación de calidad que se ocupe de sus necesidades básicas de aprendizaje y que enriquezca su vida. Si bien la educación inclusiva presta especial atención a grupos vulnerables y marginados, su fin es desarrollar al completo el potencial de todo individuo y no sólo el de grupos específicos. Conviene además distinguir el movimiento inclusivo como una propuesta diferente al modelo de integración educativa extendido en numerosos países occidentales.

La inclusión es un proceso, un camino que emprenden las escuelas con el fin último de conseguir progresivamente que todos sus miembros se sientan parte integrante del centro, aceptados y valorados. Por tanto, no existe una escuela totalmente inclusiva (Ainscow, 2005; Echeita y Ainscow, 2011). Por el contrario, la integración es un estado que se alcanza cuando todo el estudiantado recibe educación en el mismo lugar. Es decir, la integración lo que pretende es que el alumnado se integre con el resto de estudiantes pero no implica cambios o transformaciones profundas en las metodologías didácticas de los docentes y las instituciones educativas.

La integración sólo trabaja desde las dificultades y las deficiencias. Los alumnos y las alumnas con necesidades específicas de atención educativa son considerados una carga que hay que asumir y sobrellevar. En cambio, la inclusión no se centra en las deficiencias, sino en las potencialidades de cada estudiante, para partir de ellas y lograr que todos participen. En la integración lo que importa es que todos los niños estén en un mismo lugar, que tengan igualdad de acceso. Se consigue con el hecho de estar sin más, esto es, como si tener alumnado discapacitado en un aula ya fuera por sí solo un elemento pedagógico de valor. Obviamente, la inclusión requiere no sólo estar, sino participar de manera efectiva, cambiando mentalidades en la práctica docente y fomentando el uso de estrategias metodológicas más cooperativas, especialmente transformadoras del contexto educativo. Es más, esta participación no afecta sólo a estudiantes, sino que se extiende también al profesorado, familias, personal del centro y a toda la sociedad (Parrilla, 2002).

No se trata de planificar mejoras para unos grupos de estudiantes y olvidarnos de otros grupos, sino que todos deben participar, promoviéndose espacios de autenticidad en las relaciones humanas que se dan entre los propios estudiantes, y entre los estudiantes y los docentes. De ahí que en el proceso hacia la inclusión sea fundamental ir eliminando las barreras que impiden o dificultan esa participación interactiva (Echeita y Ainscow, 2011). 
Si como docentes nos planteamos que la atención de determinados estudiantes puede entorpecer o retrasar el avance de estudiantes "normales", estaremos trabajando desde una lógica claramente excluyente. Desde un modelo inclusivo, hemos de preguntarnos cómo atender a todo el alumnado, no cómo atender a unos u otros. La inclusión requiere un compromiso profesional, mientras la integración simplemente se asume y no se cuestiona. La inclusión es también una cuestión de valores, de opción cultural, social y política (Booth, 2009). Conlleva una modificación de la actitud y del discurso que ha de tener su reflejo en la práctica. Así, supone un cambio progresivo en la forma de concebir la diversidad y la práctica cotidiana en las aulas, que debe ser más democrática y colaborativa, fomentando las relaciones entre la escuela y la sociedad.

En pocas palabras, podríamos decir que, ante la diversidad, el foco de atención ha de trasladarse desde el alumno al contexto. En lugar de fijarnos en las peculiaridades de nuestro alumnado, hemos de preocuparnos para que nuestro centro educativo sea capaz de responder a las necesidades de ese grupo de estudiantes, y eso ha de reflejarse en el pensamiento del profesorado y en las prácticas educativas, que han de adaptarse al alumnado y no al revés (Durán y Giné, 2011). Este cambio de paradigma requiere un esfuerzo notable. He aquí el desafío que la escuela inclusiva propone a la formación de nuestros docentes.

Podemos identificar varios modelos de formación del profesorado en función de cómo conciban dos elementos clave de la identidad profesional: el conocimiento profesional (teórico-práctico) y el grado de autonomía en el trabajo. Así, podemos distinguir entre el enfoque práctico-artesanal, el enfoque técnico-academicista, y el enfoque reflexivo o de investigación en la acción (Pérez, 1998):

a) El enfoque práctico-artesanal. El conocimiento acerca de la enseñanza se genera en un proceso de ensayo y error y es transmitido de generación en generación. Así, el aprendizaje del conocimiento profesional por parte de los nuevos docentes se reduce a un proceso de socialización en la cultura de la escuela. Este enfoque supone la reproducción de los hábitos escolares dominantes, por lo cual tiene un carácter marcadamente conservador que deja escaso lugar a la innovación. Los docentes disfrutan de escasa autonomía pues su actividad se limita a perpetuar el legado cultural heredado. Este enfoque refuerza el pensamiento pedagógico vulgar, ya que el proceso de formación no se usa para que el docente cuestione críticamente las prácticas pedagógicas que ha 
presenciado en su vida como estudiante, sino para que las incorpore a su repertorio profesional.

b) El enfoque técnico-academicista. Aquí el conocimiento profesional no surge de la práctica del docente, sino que es elaborado por especialistas externos que dictan las normas de intervención educativa. El trabajo del docente no es, por tanto, producir conocimiento experto 0 al menos dominarlo. Su labor consiste en ejecutar sistemáticamente las directrices que se le indican en el currículum oficial. Así, la autonomía del docente es poca dado que el número de decisiones que el docente debe tomar se reduce al mínimo. El periodo práctico de formación se destina a que los nuevos docentes apliquen en la práctica los conocimientos técnicos que han estudiado en la teoría. Este enfoque ignora la dimensión ética y política de la intervención educativa, reduciéndola a un conjunto de decisiones técnicas sobre qué estrategias o recursos son los más adecuados para conseguir determinados objetivos de enseñanza.

c) El enfoque reflexivo. La investigación en la acción. Este enfoque parte de la premisa de que los docentes trabajan en una realidad social muy compleja en la que los problemas no pueden resolverse aplicando técnicas o recetas preestablecidas. El conocimiento experto siempre es emergente, pues se genera en el propio escenario, a partir de la investigación sistemática sobre un contexto concreto en la que se utiliza como herramienta conceptual todo el bagaje intelectual de la cultura crítica. Así, la reflexión sobre la práctica apoyada en el conocimiento científico permite al docente comprender mejor su labor y mejorarla. Por tanto, el conocimiento profesional se genera de forma dialéctica a través de una conversación permanente entre teoría y práctica. Dentro de este enfoque, la autonomía profesional es relativa al proceso de elaboración del conocimiento práctico, que siempre es provisional y parcial y que queda legitimado en tanto en cuanto el docente sea consciente de su relatividad contextual y del carácter político y ético del mismo. El proceso de formación del docente, de acuerdo con este enfoque, ha de destinarse a conocer el conocimiento crítico, depurado por el escrutinio de la comunidad científica, y a aprender a usarlo como herramienta de apoyo para la reflexión sobre la práctica pedagógica dominante y sobre el conocimiento pedagógico vulgar propio adquirido como resultado de su experiencia vital como estudiante. El docente se convierte así en un intelectual. 
Tras un análisis de los modelos de formación expuestos, parece evidente que la escuela inclusiva demanda profesionales reflexivos capaces de tomar decisiones para adaptar sus aulas a las necesidades de su alumnado. Sin embargo, los modelos artesanal y técnico siguen teniendo una gran vigencia en nuestro sistema educativo y suponen un gran escollo para el desarrollo de la educación inclusiva.

La situación actual en España necesita lo que Giroux (1997) denomina "profesores como intelectuales transformativos". El intelectual transformativo es el docente que analiza su práctica con el fin de crear espacios donde todos y todas tengan las mismas posibilidades, tanto dentro de la escuela como cuando salgan de ella. Es un profesional comprometido con la lucha por una escuela sin exclusiones. Si queremos transformar la realidad educativa para que se ajuste a los parámetros inclusivos, el camino es dar al profesorado la oportunidad de tomar sus propias decisiones para ajustarse al contexto en el que se mueven. Y esto nos lleva a la conclusión de que no es posible contemplar propuestas de innovación y mejora en la escuela sin proponerse tales mejoras en la formación del profesorado, que es quien puede y debe protagonizar de un modo activo dicha innovación. Un profesorado pobremente formado no estará en condiciones de asumir la responsabilidad que supone tomar decisiones sobre la acción educativa inclusiva y tampoco podrá valorar a posteriori la conveniencia de dichas decisiones para mejorarlas. Pero, al mismo tiempo, el profesorado no se verá en la necesidad de formarse si no siente que la responsabilidad de la acción educativa es suya. Por tanto, formación y autonomía van de la mano (Almenta y Muñoz, 2007).

Hablar de formación del profesorado, así pues, es hablar de autonomía. Autonomía para pensar, reflexionar y decidir el devenir de la acción educativa. En palabras de Stenhouse (1998), la planificación es la herramienta más potente de formación del profesorado. Cuando el docente se enfrenta a la tarea de pensar cómo dar respuesta a las necesidades de su aula (Salinas, 1994), es cuando está planificando y se está formando como docente. Por tanto, ésta es la planificación a la que nos referimos cuando hablamos de herramienta de formación del profesorado, herramienta que no puede desarrollarse ajena al espacio que le brinda la autonomía.

Ahora bien, no podemos plantear una formación reflexiva y crítica sin conocer las ideas, pensamientos y concepciones que tiene nuestro alumnado en el contexto universitario. Ideas y concepciones determinadas por varios factores que sólo mencionamos 
sin profundizar en ellos: biografía escolar, rasgos de personalidad, experiencia formativa universitaria, contraste entre la teoría de la universidad y la visión práctica del período de formación de prácticas externas en centros educativos, ambiente sociocultural, condicionantes económicos, etc.

\section{La percepción del alumnado universitario de la carrera de educación especial ante el concepto de educación inclusiva}

Los datos que aquí presentamos forman parte de un estudio de investigación desarrollado con el alumnado de la asignatura denominada "Bases pedagógicas de la Educación Especial”, de la titulación de Maestro, especialidad Educación Especial, de la Facultad de Ciencias de la Educación de la Universidad de Málaga (Andalucía, España), en el curso académico 2011/2012, en concreto durante el segundo cuatrimestre, de febrero a junio de 2012.

Este estudio cualitativo pretendía conocer y comprender el pensamiento pedagógico de este alumnado sobre el sentido y significado de la educación especial, para ir generando un nuevo concepto de educación inclusiva abierto y crítico. En este estudio, participaron 70 alumnos y alumnas, de los 104 matriculados en dicha materia. Como instrumento de recogida de datos cualitativos empleamos la entrevista semiestructurada y el autoinforme. Lógicamente, partíamos de ideas previas tratadas en una sesión presencial específicamente destinada a cuestionar el concepto actual de la educación especial. De ahí surgieron tanto las preguntas como las dimensiones conceptuales, instrumentales y éticas de la experiencia que presentamos. En este sentido, la primera idea que podemos plantear es que muchos o del estudiantado estaba convencido de que el concepto de educación especial no podía limitarse a una perspectiva meramente técnica o de apoyo específico a un tipo de alumnado con necesidades educativas especiales, sino que había que generar una nueva definición a partir de una visión social y pedagógica más abierta e igualitaria:

Bajo mi punto de vista, la educación especial es un concepto mal identificado por parte de la sociedad debido a la gran pluralidad terminológica que conlleva. Sin embargo, me atrevo a decir que es un proceso de enseñanza donde se busca obtener la igualdad hacia las personas con necesidades con el empleo de ayudas o métodos dedicados a conseguir un progreso equivalente en todas las diferentes áreas de desarrollo del sujeto, así tanto en aspectos físicos y cognitivos como en los personales. (Alumno ํㅜ 1) 
Resulta muy interesante la crítica que este alumnado de la titulación de Educación Especial realiza a otro alumnado de otras especialidades en la misma Facultad. Esto lo podemos atribuir a la percepción que tienen sobre la escasa formación en temas de atención a la diversidad que reciben los futuros docentes especialistas o las propias alumnas y alumnos de Educación Primaria. En este punto, algunas alumnas apuntan a que la propia Educación Especial es desconocida para otros estudiantes universitarios.

...a mi también me parece que el término de educación especial es un término muy desconocido y por ello se ve desde un punto de vista apartado de la realidad y de lo que la sociedad considera como normal. Ahora bien, los especialistas en el campo de la educación especial como seremos nosotros en el futuro somos los encargados de concienciar a la sociedad de que la educación especial es un camino más para hacer llegar a nuestro alumnado a conseguir los mismos objetivos; es un camino especial para llegar hacia los mismos objetivos pero por ello no le tenemos que poner trabas ni etiquetas ya que todas las personas aprendemos de forma distinta independientemente de si necesitamos NEE o no. (Alumna no 4)

Otra idea sugerente y muy importante tiene que ver con el cambio de denominación de Educación Especial a Educación inclusiva. En efecto, muchas alumnas se cuestionaban por qué estudiaban Educación Especial, qué era realmente, y qué tenía de "especial" la formación que recibían en la facultad y la que iban a impartir como futuros profesionales de la educación.

....me gustaría compartir mi idea de Educación Especial. A mi parecer es un término que se ha quedado obsoleto, pues ¿por qué especial?, ¿porque es distinta?, ¿por qué se adapta a las necesidades del niño? ¿No tendría que ser siempre así se tenga o no una discapacidad? ya de antemano se le pone un nombre distintivo: "especial", con lo que a priori estamos diferenciando a esos niños "Ios del aula especial". Creo que los niños con discapacidad reciben una educación que se adapta a sus necesidades de esta forma se optimiza el potencial individual de cada uno, llegando en muchos casos a los mismos objetivos que los niños sin discapacidad. En los casos que no se llega a esos objetivos, se marcan otros, igual de importantes que los anteriores con la diferencia de que son asequibles a ellos. ¿No creéis que tendría que ser así con todos los niños? Por todo esto, ¿para cuándo el cambio de nombre? (Alumna nํ5) 
Otro alumno defiende la idea de que la Educación inclusiva debe ser una apuesta pedagógica y social decidida por promover una ciudadanía crítica y autónoma, donde la diversidad funcional no sea vista como una carga sino como una oportunidad para las personas y para los contextos, sean educativos, sociales, culturales, y, por supuesto, laborales.

....es que debemos como futuros maestros que somos detectar el nivel de cada uno de nuestros estudiantes a nivel motor, cognitivo...y saber cuáles son las necesidades que presentan cada uno de ellos de esta manera podremos hacer una enseñanza adaptada, personalizada e individualizada para sacar el máximo potencial de todas sus habilidades. Por ello pienso que la formación del profesorado es crucial para poder atender a estos niños y tener los conocimientos específicos y recursos necesarios para atender las diversas necesidades de ellos. Nosotros debemos de preparar a nuestro alumno para que por el mismo sea una persona autosuficiente, que se sienta útil dentro de la sociedad en la que vivimos y debemos de prepararlo de cara a un futuro con respecto a la vida laboral. (Alumno nํ21)

En esta misma línea, otro alumno añade que la Educación inclusiva debe plantearse no tanto como una atención educativa de carácter personal o individual, sino por el contrario, que debe ser una educación que opte decididamente por promover una convivencia y unas pautas didácticas de aprendizaje de índole cooperativas. Es decir, todas las personas precisamos una educación inclusiva en la medida que todos aprendemos de todos y con todos. De tal manera, el sentido y orientación que adopta el proceso de enseñanzaaprendizaje determina la propia concepción pedagógica de la educación inclusiva. Así se expresaba este alumno:

En mi opinión, la educación "especial" me parece un término erróneo, ya que parece hacer referencia siempre a una atención individualizada, atendiendo siempre a las capacidades y habilidades concretas de cada uno de los estudiantes, a sus necesidades y características. $Y$ digo yo: ¿acaso no es esa la educación que convendría impartir en todos los casos, y no solo con respecto al alumnado con algún tipo de dificultad o trastorno? ¿No es cierto que todos y todas tenemos dificultades en algún momento de los procesos de enseñanza-aprendizaje? Para mí, la educación inclusiva trata de desarrollar las habilidades de los sujetos con dificultades para 
hacerlas potencialmente óptimas, de manera que dichas personas puedan llevar a cabo el ejercicio de capacidades básicas en su día a día. No obstante, opino que dichas competencias no solo deberían aplicarse al aspecto más propio del término, ya que todos/as precisamos de una "educación especial", una atención especializada que busque el desarrollo de nuestras habilidades al máximo. (Alumno nํ33)

Para mucha de la población estudiantil entrevistada la idea de la Educación inclusiva no se corresponde con la realidad que han visto en el Prácticum y la que ellos han vivido en su propia biografía escolar. Ante esta idea, son muchas las voces de quienes piensan que esta concepción pedagógica tiene mucho de utopía. Ahora bien, la utopía no es algo negativo en la formación, sino todo lo contrario, un incentivo netamente motivador para profundizar aún más en la necesidad de formarnos cooperativamente. En este sentido, una alumna critica una vez más el uso del término especial y defiende la apertura y significado sociocultural de la concepción pedagógica inclusiva.

Creo que buscar una definición concreta para educación especial resulta muy difícil, cuando no imposible. Cada uno de nosotros utilizamos términos intentando dar respuesta a este enigma, pero todos nos acercamos y/o nos alejamos en mayor o menor grado, según las lentes con las que se mire. Desde las mías, educación especial puede resultar incluso una utopía. En sí la educación se suele ver como algo dirigido a.... (alumnado) y en el caso de educación especial, ésta es dirigida a....... (alumnado con....) ¿Educación especial y educación siguen un camino paralelo, o son la misma vía? Partiendo de las leyes donde se hace mención a la inclusión, y entendiendo que inclusión abarca a todo el grupo de alumnado, entonces, resulta obvio que educación especial no puede dirigirse solo a alumnado con.... sino, que va orientada a todo el grupo de alumnado. Pero, entonces ¿existe diferencia entre educación y educación especial? Yo no lo tengo todavía claro, pero si nos ceñimos a las definiciones podemos aparte de volvernos locos, deducir que algo aquí nos sobra.... Como ya he dicho, no observo diferencia entonces entre educación y educación especial... tal vez el término de educación sea bastante pobre como para abarcar la gran diversidad de las aulas de hoy día, o tal vez debería asumir los papeles que se le asignan al concepto de "educación especial. (Alumna ํㅜ 49) 
Una idea relevante en la emergencia de la construcción de la Educación inclusiva, aquella educación que considera que no se debe tratar terapéuticamente a los niños con discapacidad fijándose en sus deficiencias, sino por el contrario, transformar los contextos educativos, cambiar mentalidades y atender de una manera más abierta, cooperativa y competencial a todo el alumnado; es precisamente que los estudiantes universitarios tienden a la confusión conceptual y terminológica, sobre todo con la idea de integración versus inclusión, y a veces con la atención a la diversidad. Así, algunas alumnas planteaban que las cuestiones terminológicas no son importantes porque no observaban diferencias semánticas significativas entre integración e inclusión. En todo caso, esto ha sido un punto polémico del debate del propio alumnado, ya que sí existen diferencias conceptuales significativas en el marco de la Educación inclusiva, aunque lo que realmente lo demuestra no son los modelos o teorías, sino las propias prácticas docentes. En este sentido, es posible que las prácticas observadas en el periodo de prácticas, o algunas de ellas, no se ajusten al paradigma de Educación inclusiva sino al de una Educación Especial. Por tanto, si bien a nivel teórico se defiende la Educación inclusiva, lo que se lleva a cabo en la práctica escolar es Educación Especial. Este choque o disonancia de significado pedagógico es algo que desborda a veces al alumnado:

He de confesar que la pluralidad de términos me abruma y no pocas veces he acabado desorientado, aún así reconozco el valor positivo de la terminología siempre y cuando traiga consigo elementos pragmáticos consecuentes y coherentes, es decir, que aporte algún cambio efectivo en el significado que hay detrás de, de lo contrario no será más que una taxonomía inútil. Eso me lleva a pensar en el concepto de educación especial y lo que conlleva pero, ¿qué entendemos cuando complementamos el término educación con especial? ¿se trata de poner al alcance de determinadas personas recursos (más, diferentes, alternativos, es igual) para potenciar su desarrollo personal? ¿tal vez se entiende como una manera de hacer distinción (positiva o negativa) entre unas personas $y$ otras? Me parece importante matizar este punto, puesto que diariamente utilizamos expresiones sin llegar a vislumbrar con claridad qué estamos poniendo en juego al expresarlas, yo el primero. Por tanto, para mí la educación especial NO es una forma o rama subordinada de la educación, sino un proceso educativo en sí mismo que persigue el desarrollo global de la persona (independientemente de si posee o no discapacidad), apuesta por sus posibilidades $y$ 
lucha por su derecho de autodeterminación, entonces, ¿difiere tanto en sus fines como para establecer una división tan acusada entre educación y educación especial? (Alumno $\mathrm{n}^{\circ}$ 32)

El alumnado pone de manifiesto que esa disonancia de la que hablamos entre la definición de Educación Especial que encuentran en las aulas educativas y la definición que ellos creen correcta, está estrechamente relacionada con la cualificación de todo el equipo de profesionales que trabajan en un centro educativo para responder mejor a las necesidades de todo el alumnado y maximizar sus posibilidades de desarrollo a todos los niveles.

Al plantearme la idea de realizar mi propia definición de Educación Especial, me han surgido dos interrogantes: ¿debo definir (según yo) la Educación Especial existente en algunos (por no decir mayoría) centros de hoy? o bien ¿debo definir lo que considero que debe ser la Educación Especial? Según la primera, considero que muy a nuestro pesar podríamos aplicar algunas de las definiciones leídas en clase, $y$ conforme a la segunda podría decir que bajo mi punto de vista debemos luchar por una Educación "Especial", que implique no solo el conocimiento de determinados profesionales, sino a todo el equipo educativo con el objetivo de estar mejor preparados para enfrentarse a las necesidades que presentan la diversidad de alumnado existente en nuestras aulas, de este modo se les podría ofrecer a través de un trabajo interdisciplinar, a cada alumno/a, vías alternativas de aprendizaje que favorezcan el desarrollo de sus potencialidades, conforme a sus propias características individuales y de su entorno, trabajando el desarrollo de sus aptitudes físicas, intelectuales, comunicativas, emocionales y sociales. (Alumna $n^{\circ} 63$ )

Es recurrente entre el alumnado la concepción de educación especial como educación para todos y todas. Además, destacan que la educación especial no sólo ha de centrarse en aspectos puramente cognitivos, sino que, como parte del proceso educativo integral de las personas ha de preocuparse del desarrollo de habilidades sociales, habilidades motoras, etc. Aún así, es significativo señalar que el uso del término "integración" está tan arraigado en la cultura escolar que el alumnado habla de "fomentar la integración" cuando parece que la descripción del término está más cercana al concepto de inclusión. 
En mi opinión la educación especial no debe de centrarse sólo en las personas con algún tipo de deficiencia mental, sino en muchas otras personas que necesitan esa ayuda especial. Como comentaba María, las personas superdotadas necesitan la esa ayuda especial para poder relacionarse con el entorno que le rodea que, en mi opinión, es el mayor problema que presentan. La educación especial no tiene por qué estar únicamente centrada en potenciar competencias cognitivas sino enseñar habilidades sociales, habilidades motoras, etc. La educación especial debe de centrarse a toda persona con problemas para la integración social, retraso mental, retraso motor, etc. Nosotros como futuros docentes de esta carrera que estamos estudiando debemos de potenciar al máximo las capacidades de este alumnado y siempre intentar trabajar en el aula ordinaria con los demás compañeros, esto fomentará la integración con los demás. A demás de adaptarle los materiales y materias educativas para potenciar al máximo su capacidad de desarrollo. (Alumna no 47)

Gran parte del alumnado reconoce que no se había enfrentado antes a la tarea de definir el término educación especial, pero cuando lo hacen llegan a la conclusión de que la educación especial ha de ser la menos especial (por peculiar o infrecuente) de las educaciones. Es significativo que los propios estudiantes de magisterio apunten hacia la formación del profesorado como uno de los cambios necesarios para generar un contexto escolar con igualdad de oportunidades.

La verdad es que aún no tenía nada pensado, no sabía muy bien cómo puede definirse la terminología "educación especial", pero no dejan de llegarme emails al correo y mi conciencia me dice que me ponga ya a hacer este ejercicio. Permitidme una pequeña reflexión. Educación especial, son dos palabras, obviamente, pero que no se asemejan mucho el significado que tiene la una con la otra. Educar, sacando el diccionario de latín que me compré en el bachiller de humanidades, viene dado de la palabra educere que significa: educar, guiar, conducir, formar, instruir, pero hoy en día, tiene otro significado dentro de la escuela: transmitir conocimientos, valores, costumbres y formas de actuar, a través de acciones, sentimientos y actitudes. Un proceso de concienciación moral, cultural y conductual. Proceso de socialización. Se fomenta el respeto. Entonces... mi primera pregunta que me ayuda en esta reflexión es ¿eso no son los objetivos generales que nos vamos a marcar para trabajar con nuestros futuros 
niños y niñas? Especial, creo que es aquello poco común, singular, peculiar, etc... Me cuestiono a mí misma y me pregunto, el niño que recibe apoyo ¿es un niño peculiar? (Alumna $n^{\circ}$ 51)

El alumnado reflexiona sobre las connotaciones peyorativas que el adjetivo "especial" tiene dentro del campo educativo y termina concluyendo que todos y todas somos especiales porque en algún momento necesitamos apoyos que van más allá de lo ordinario o lo común. De ahí que especial deba ser entendido como un reconocimiento de la diversidad y no como una situación negativa o lacra perturbadora.

Empezar diciendo, que cuando leemos "especial" en cualquier contexto, o nos referimos a algo "especial" en nuestra vida, nunca toma el matiz que toma en la "educación". Aquí cuando nos referimos a "especial", al igual que en otros contextos, es "diferente", pero sólo en la educación o en el ámbito educativo, "especial" equivale o es sinónimo de: malo, peor, raro, siempre en tono peyorativo, marginal, excluyente. No es así cuando queremos un "viaje especial" o "un vestido especial"... ¿Por qué esto ocurre en educación? ¿Por qué tienen que salir del aula, por qué no siguen un curriculum ordinario, por qué no siguen el ritmo, por qué les cuesta relacionarse, por qué no participan, por qué no son autónomos y mil cosas más? Pero cada uno de nosotros en algún momento de nuestra vida hemos necesitado o necesitaremos una atención especial, una explicación especial y no por ello se nos mira raro. Es cierto que estas personas necesitan esa "especial" durante toda la vida, pero ¿por qué especial?, porque no es lo común. (Alumno nํ53)

El alumnado es consciente del lastre que el concepto de Educación Especial ha de soportar viene en gran parte dado por la herencia del sistema educativo anterior a la LOGSE (1990), en el que existían dos itinerarios paralelos, siendo éstos el ordinario y el especial. De ahí que se defienda la Educación Especial como un sistema único que ha de responder a las necesidades de todo el alumnado y que ha de tener como misión la inserción de las personas en los contextos sociales y la transformación de dichos contextos para hacerlos más humanos.

Considero que todavía queda en la práctica educativa bastantes restos de lo que en su día la LOGSE quiso eliminar: la existencia de dos sistemas educativos paralelos, el 
general u ordinario y el especial. Dándose una división educativa que, a mi parecer, lo único que hace impedir la construcción de una escuela inclusiva. Pues, ¿cómo podemos defender los principios de equidad e igualdad de oportunidades, si lo primero que se hace es clasificar al alumnado en dos grupos: "normales" y "no normales" a lo que les corresponde un tipo de educación concreta? Incluso me atrevería a cambiar el verbo clasificar por cribar, porque siquiera creo que se agrupe, sino que se selecciona. Para mí, la educación especial compondría un único sistema educativo capaz de responder a las necesidades educativas de todos y cada uno de los estudiantes. La expresión "educación especial" puede referirse tanto a una actividad social (y a una práctica profesional) como a una disciplina científica; en cualquier caso, el referente de esta actividad son las personas con necesidades educativas especiales. (Alumno n55)

Se entiende que el término "especial" ha sido usado para justificar prácticas escolares discriminatorias, y por eso es que se considera que debemos deshacernos del adjetivo especial y recordar que la educación ha de ser educación para todos y todas, con independencia de las necesidades educativas de cada cual.

Considero que ésta definición es muy completa y me gusta mucho, incluso sería la que yo emplearía o muy similar, pero tanto para el concepto de educación especial como para el de educación. Por tanto me reitero con la idea (personal) de que algo en éste planteamiento sobra... Como bien indica Jorge P., ¿Qué significa la coletilla "especial"? Como hablamos en otra asignatura, ¿creéis que educación especial responde a alumnado con necesidades educativas especiales? (Alumna ํㅡ 56)

Merece la pena destacar que el alumnado es consciente de la necesidad de que la educación sea un proceso holístico que ayude a las personas a desenvolverse con igualdad de oportunidades en todos los ámbitos de la vida, ya sea el formativo, el profesional o el social y personal. $\mathrm{Y}$ este proceso educativo ha siempre de desarrollarse en el contexto de diversidad que caracteriza a cualquier grupo humano.

Desde mi punto de vista y debido a la práctica realizada en clase puedo decir que considero la Educación Especial como una educación que busca integrar a las personas de una manera escolar, laboral y social. Ofreciéndole así una igualdad de oportunidades a niños y niñas a pesar de la diversidad que todos y todas tenemos. Es 
necesario resaltar que todos y todas somos diferentes por lo que siempre tenemos la diversidad presente. Por lo que tenemos que potenciar fomentar un desarrollo global trabajando de manera interdisciplinar adaptándonos a las necesidades individuales que todas las personas necesitamos fomentar. Centrándose de esta manera en el niño o niña y su entorno. (Alumna $n^{\circ} 61$ )

En último lugar, pero no por ello menos importante, hay que destacar la necesidad de que toda la sociedad, y no solo los profesionales del ámbito educativo, aprendan a convivir con personas con diversidad funcional, huyendo de un trato de pena o caridad para construir un proceso de socialización igualitaria y plena.

Considero que la Educación Especial, al igual que cualquier tipo de educación, es un proceso de concienciación, socialización e incluso identificación, que toda persona debería tener en su repertorio conductual y cognitivo; a la hora de relacionarse con personas que presenten alguna característica diferenciada, o lo que es lo mismo, especial. Por tanto, la Educación Especial no sólo debería formar parte del gremio dedicado a esa población. Además opino que la Educación especial no debería ser igual a "trato compasivo" (mal entendido claro), pues esa actitud ya estuvo muy patente y sin resultado positivo durante muchos años. Me refiero a un trato "igualitario" de manera que estas personas puedan desenvolverse satisfactoriamente en su entorno. Aunque esto último no resta importancia a las barreras que actualmente y por desgracia aun existen para estas personas. (Alumna $n^{\circ}$ 65)

\section{Propuestas para una formación inclusiva del profesorado de educación especial: un cambio de mirada}

Del mismo modo que estamos convencidos que un modelo meramente técnico de formación docente no preparará a los profesionales de la educación para afrontar con garantías de éxito los retos de la escuela inclusiva, no pretendemos en este trabajo dar recetas sobre cómo ha de diseñarse la formación de los estudiantes universitarios que estudian Ciencias de la Educación. No obstante, aun siendo conscientes de que nuestra experiencia está ligada a un contexto específico, creemos que exponerla y someterla al escrutinio de la comunidad científica puede servir a un doble fin. Por un lado, dar ideas a otros docentes sobre cómo diseñar la formación de los estudiantes de asignaturas de 
Educación inclusiva en el contexto universitario. Y por otro, hacer un ejercicio de análisis y revisión de nuestra propia mentalidad pedagógica sobre formación inclusiva a nuestro alumnado, futuros maestros de escuelas inclusivas en la sociedad del siglo XXI.

Si tal y como hemos expuesto anteriormente el docente de la escuela inclusiva necesita conocer la naturaleza de la cultura que enseña, ser consciente de lo que significa una escuela inclusiva, ser crítico, autónomo y responsable, saber analizar, y en base a todo esto tomar decisiones, resulta obvio que necesita una formación que se mueva dentro del paradigma reflexivo. Así, compartimos con Pérez (1998) la premisa de que la formación del pensamiento práctico (o teoría sobre la práctica) del futuro docente ha de desarrollarse a través de un proceso dialéctico de reconstrucción del conocimiento pedagógico vulgar que se adquiere a través de la experiencia como estudiante. El paso por el sistema educativo hace que el estudiante asuma acríticamente las estrategias metodológicas que ha presenciado, y la forma de cuestionar estas concepciones es contrastarlas con el conocimiento científico, para que se produzca el conflicto cognitivo que las transforme.

Partiendo de esta base conceptual, nos planteamos que cualquier asignatura con desarrollo de competencias en materia de educación inclusiva, así como aquellas específicas que se vinculen a una mención de educación inclusiva se conciban como laboratorios pedagógicos creativos donde usar el conocimiento teórico sobre la educación inclusiva como herramienta pedagógica crítica para cuestionar las propias concepciones y avanzar hacia una cultura de la diversidad entre el propio alumnado universitario. El hecho de que los estudiantes hayan realizado prácticas en centros educativos sería un elemento muy interesante, ya que va a propiciar el contraste de la teoría no sólo con la experiencia de los estudiantes en su vida académica, sino también con la realidad que han conocido tras su paso por los centros educativos como estudiantes en prácticas.

En este punto, somos defensores de la necesidad de que las prácticas se desarrollen de manera paralela al propio curriculum universitario en todos los años y cursos. La teoría y la práctica deben estar en un permanente diálogo fluido y crítico que esté al servicio del aprendizaje compartido y el crecimiento personal y profesional de todo el alumnado universitario de educación. Lógicamente esta propuesta debería ser consensuada por todos los agentes educativos y exigen una alta coordinación entre instituciones tales como las facultades de ciencias de la educación, las delegaciones provinciales de educación y los propios centros de educación infantil y primaria. 
Desde nuestro punto de vista, consideramos que cualquier propuesta formativa en educación inclusiva debe atender a un conjunto complejo, dinámico, flexible y versátil de ejes temáticos que deben ir permanente complementados por la propia emergencia y negociación entre profesorado y alumnado universitario. Esto supone un cambio de mirada desde la discapacidad a la competencia, desde los conocimientos de las diferentes discapacidades a las competencias procedimentales y actitudinales para promover en las escuelas metodologías inclusivas y, por tanto, cooperativas y facilitadoras de aprendizaje relevante. Estos serían los núcleos temáticos ineludibles para una óptima formación inicial del profesorado en materia de inclusión social y educativa:

$\checkmark$ De la exclusión a la inclusión. Precisiones terminológicas. Origen y sentido de la educación inclusiva.

$\checkmark$ La dimensión ideológica, política y ética de los procesos de planificación, desarrollo y evaluación de las prácticas inclusivas.

$\checkmark$ Modelos de formación de docentes para la escuela inclusiva. El docente como intelectual reflexivo.

$\checkmark$ Ciudadanía y democracia: la inclusión como ejercicio democrático.

$\checkmark$ La educación intercultural: fundamentos y competencias.

$\checkmark$ La diversidad funcional: desde el modelo de minusvalía al modelo de diversidad.

$\checkmark$ Género y diversidad afectivo-sexual.

$\checkmark$ Inclusión, TIC y redes sociales. Alfabetización digital.

$\checkmark$ La deprivación sociocultural.

$\checkmark$ El acceso a la cultura y al ocio de los colectivos más vulnerables.

$\checkmark$ Estilos de aprendizaje. La teoría de las inteligencias múltiples.

$\checkmark$ Estrategias organizativas y curriculares para la inclusión.

$\checkmark$ Familias y escuela. Modelos de participación y colaboración.

$\checkmark$ El marco legal en materia de atención a la diversidad: posibilidades y limitaciones.

$\checkmark$ Modelos y experiencias prácticas de escuelas inclusivas.

Todos estos temas se tratan a partir de la lectura de textos y su posterior análisis en clase a través de preguntas que versan sobre el contenido de los mismos y que pretenden conectar y contrastar el desarrollo conceptual de los textos con la realidad educativa que conoce el alumnado. También se utiliza material audiovisual (viñetas, películas, anuncios, 
canciones, noticias de prensa, etc.) como punto de partida para la reflexión, del mismo modo que se invita a profesionales de la educación en activo para que compartan su experiencia en materia de educación inclusiva. Además, todos estos contenidos se utilizan con el objetivo de que el alumnado se forme en las siguientes competencias:

- Vivencia de un modelo de enseñanza que desarrolle en el futuro docente el trabajo autónomo y la responsabilidad reflexiva, conectando los aprendizajes teóricos y prácticos a través de la reflexión, tomando conciencia de las teorías que aplica y confrontando lo que podría hacer con su posición ideológica, para que no existan contradicciones entre ambas.

- Conocimiento del alumnado y del currículum y normativa oficial, que permitan adaptar las directrices curriculares a las necesidades del alumnado.

- Manejo de metodologías y estrategias de inclusión, como pueden ser el aprendizaje cooperativo, las tutorías de iguales, el diseño multinivel, flexibilización de tiempos y espacios, etc.

- Formación en selección de recursos, considerando la adecuación al contexto y su conveniencia de acuerdo con la planificación educativa, pilar fundamental de un modelo responsable de actuación.

- Familiarización con la metodología de investigación-acción que permita la reflexión sistemática sobre la práctica.

- Valoración del trabajo docente en equipo y las comunidades de aprendizaje como herramientas de aprendizaje profesional, concibiendo la figura del profesorado de apoyo y de los orientadores como profesionales con los que se comparte la responsabilidad de la toma de decisiones educativas en lugar de cómo "solucionadores de problemas especiales".

- Creación de redes de trabajo que sobrepasen las fronteras del centro para comentar, intercambiar y apoyar propuestas inclusivas y así mejorar la acción.

- Compromiso de toda la comunidad educativa para la construcción de una escuela inclusiva como espacio de convivencia y celebración de la diversidad.

\section{Conclusiones}

Llegados a este punto queremos subrayar la importancia de la formación en educación inclusiva para el joven profesorado que se está formando en las Facultades de Ciencias de 
la Educación de nuestros contextos, lo cual va a tener amplias repercusiones sociales desde el punto de vista de la cohesión y el bienestar para las jóvenes con discapacidad y sus familias, así como para la sociedad en su conjunto.

Formar en inclusión educativa es formar en convicciones para la vivencia democrática, justa, digna y solidaria de vidas compartidas que viajan juntas en un mundo lleno de incertidumbre social, cultural y económica. Esto es una conclusión que se desprende de la experiencia narrada, y es que el alumnado universitario no sólo quiere conocer aspectos concretos de ciertas diversidades funcionales sino modos de trabajar, modos de funcionamiento pedagógico que les sirvan para construir inclusión en sus aulas. Son plenamente conscientes de que sus estrategias didácticas deben orientarse hacia el trabajo cooperativo y la no exclusión del alumnado.

Por todo ello, y sin perder de vista la importancia de la dimensión emocional en la educación, la formación inclusiva del profesorado debe ser intercultural y debe ser crítica en la medida en que las demandas sociales van en la línea de la multiculturalidad social y la necesidad de responder a las desigualdades sociales. Es imprescindible la formación inclusiva de este alumnado, futuro profesorado de apoyo a la integración de las aulas andaluzas y españolas en términos de cooperación y confianza. Esto es un elemento fundamental para comprender que los agentes educativos pueden y deben propiciar una convivencia inclusiva e intercultural que contemple la necesidad de pasar de una diversidad cultural ya conocida a una cultura de la diversidad por conocer. $Y$ es que, compartimos plenamente la idea de que "una educación inclusiva sólo es viable si se tejen amplias y sólidas redes de colaboración e interdependencia de todos los niveles y entre todos los actores implicados" (Echeita y Otros, 2004, p. 50). Esto significa que es necesario abrir la educación a la comunidad y la comunidad a una educación que debe dirigirse a todas y a todos sin ningún tipo de excepción.

Los principios pedagógicos de la convivencia inclusiva son los mismos que construyen una pedagogía moderna y equitativa basada en la confianza y en la cooperación como baluartes de un aprendizaje donde la diferencia es vista como un valor y no algo negativo que dificulta el entendimiento y el desarrollo educativo. Más bien al contrario, estamos de acuerdo con López (2004, p.115), cuando expresa que en todas las escuelas del mundo debería existir un letrero que dijera que la escuela es el escenario donde se "garantiza el despertar de la curiosidad y el deseo de aprender a cualquier niño o niña, con independencia 
de sus condiciones personales y sociales, de sus características étnicas, de género, de hándicap, lingüísticas o de otro tipo". La educación inclusiva es un viaje educativo donde el factor más importante es el deseo de hacer que suceda el cambio en nuestras mentes y en nuestras prácticas didácticas. Ese deseo de cambio es lo que estimulará la permanente necesidad pedagógica de promover la cultura de la innovación y de la diversidad, así como las prácticas didácticas potenciadoras del trabajo en equipo y el aprendizaje autónomo.

Los cambios en las escuelas que serán inclusivas en este siglo XXI serán definitivos cuando la formación del profesorado comporte cambios metodológicos, curriculares y de innovación pedagógica basados en una cultura de la diversidad como eje vertebrador de una concepción inclusiva de la educación que impregne todos los factores didácticos en las aulas universitarias de las facultades de ciencias de la educación.

\section{Referencias}

Ainscow, Mel. (2001). Desarrollo de escuelas inclusivas. Madrid: Narcea.

Ainscow, Mel. (2005). Developing inclusive education systems: What are the levers for change?, Journal of Educational Change, 6, 109-124.

Almenta, Estefanía y Muñoz, Juana. (2007). ¿Estamos formados para trabajar en una escuela inclusiva? En Actas del V Congreso Internacional Educación y Sociedad. La educación, retos del siglo XXI. Granada: Codoli.

Arnaiz, Pilar. (2003). Educación inclusiva: una escuela para todos. Archidona (Málaga): Aljibe.

Booth, Tony. (2009). El uso del Index for Inclusion en Inglaterra. En Climent Giné, David Duran, Josep Font y Ester Miquel (Coords.), La educación inclusiva. De la exclusión a la plena participación de todo el alumnado (pp. 143-160). Barcelona: Horsori.

Durán, David y Giné, Climent. (2011). La formación del profesorado para la educación inclusiva: Un proceso de desarrollo profesional y de mejora de los centros para atender la diversidad, Revista Latinoamericana de Educación inclusiva, 5(2), 153-170.

Echeita, Gerardo. (2006). Educación para la inclusión o educación sin exclusiones. Madrid: Narcea.

Echeita, Gerardo, Alonso, Pablo, Durán, David, Font, José, Marín, Nuria, Miquel, Enrique, Parrilla, Ángeles y Ainscow, Mel. (2004). Educar sin excluir, Cuadernos de Pedagogía, (331), 50-53. 
Echeita, Gerardo y Ainscow, Mel. (2011). La educación inclusiva como derecho. Marco de referencia y pautas de acción para el desarrollo de una revolución pendiente, Tejuelo, 12, 26-46.

Escudero, Juan Manuel y Martínez, Begoña. (2011). Educación inclusiva y cambio escolar, Revista Iberoamericana de Educación, 55, 85-105.

Giroux, Henry. (1997). Los profesores como intelectuales. Hacia una pedagogía crítica del aprendizaje. Barcelona: Paidós.

MECD. (2006). Ley Orgánica 2/2006, de 3 de mayo, de Educación. Madrid: Gobierno de España. Recuperado de http://www.boe.es/boe/dias/2006/05/04/

López, Miguel. (2004). Construyendo una escuela sin exclusiones. Archidona (Málaga): Aljibe.

López, Miguel. (2006). Cultura de la diversidad, cultura de la inclusión: educar para construir una escuela sin exclusiones. Actas de las XVI Jornadas Municipales de Psicopedagogía "L'Ecola que inclou". Ajuntament de Torrent, Col-lecció Hort de Trenor 18, 11-52.

Miles, Susie y Singal, Nidhi. (2009). The Education for All and the Inclusive Debate: Conflict, Contradiction or Opportunity? International Journal of Inclusive Education, 14(1), 1-15.

Parrilla, Ángeles. (2002). Acerca del origen y sentido de la educación inclusiva. Revista de Educación, (327), 11-29.

Pérez, Ángel lgnacio. (1998). La cultura escolar en la sociedad neoliberal. Madrid: Morata.

Pérez, Ángel Ignacio. (2012). Educarse en la era digital. Madrid: Morata.

Salinas, Dino. (1994). La planificación de la enseñanza: ¿técnica, sentido común o saber profesional? En Félix Angulo y Nieves Blanco (Coords.), Teoría y desarrollo del currículum (pp. 135-160). Málaga: Aljibe.

Stenhouse, Lawrence. (1998). Investigación y Desarrollo del Currículum. Madrid: Morata.

Susinos, Teresa. (2005). ¿De qué hablamos cuando hablamos de inclusión educativa?, Temáticos Escuela, 13, 4-6.

Susinos, Teresa y Parrilla, Ángeles. (2008). Dar la voz en la investigación inclusiva. Debates sobre inclusión y exclusión desde un enfoque biográfico-narrativo, REICE. Revista Electrónica Iberoamericana sobre Calidad, Eficacia y Cambio en Educación, 6(2), 157171.

Stainback, Susan y Stainback, William. (2001). Aulas inclusivas. Un nuevo modo de enfocar y vivir el currículo. Madrid: Narcea. 
UNESCO. (2005). Guidelines for inclusion: Ensuring Access to Education for All. París: UNESCO,

Recuperado

de http://unesdoc.unesco.org/images/0014/001402/140224e.pdf

UNESCO. (2009). Policy Guidelines on Inclusion in Education. Paris: UNESCO. Recuperado de http://unesdoc.unesco.org/images/0017/001778/177849e.pdf

Verdugo, Miguel Ángel. (2009). El cambio educativo desde la perspectiva de la calidad de vida. Revista de Educación, (349), 23-43. 